INPLASY

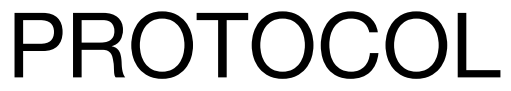

To cite: Woolley et al. The risk of osteonecrosis of the jaw (ONJ) and adverse outcomes in patients using antiresorptive drugs undergoing orthodontic treatment, A protocol for a systematic review. Inplasy protocol 202070124. doi: 10.37766/inplasy2020.7.0124

Received: 28 July 2020

Published: 28 July 2020

Corresponding author: Julian Woolley

julianwoolley@gmail.com

Author Affiliation:

King's College Dental Institute

Support: None.

Review Stage at time of this submission: Preliminary searches.

Conflicts of interest: None.

\section{The risk of osteonecrosis of the jaw (ONJ) and adverse outcomes in patients using antiresorptive drugs undergoing orthodontic treatment, A protocol for a systematic review}

Woolley, J1; Akintola, D2; Yates, J3; Calasans-Maia, MD4; Calasans-Maia, JA5; Sacco, $\mathrm{R}^{6}$.

Review question / Objective: Is there any evidence that orthodontic treatment induces osteonecrosis of the jaws (ONJ) in patients treated with antiresorptive drug therapy? -Population: patients previously or under antiresorptive drugs and orthodontic treatment; -Interventions: any orthodontic treatment; -Comparison: patients undergoing orthodontic therapy not subjected to bisphosphonate therapy (risk assumed as negligible); -Outcome: state of knowledge regarding the risk of medication-related ONJ as it refers to type of antiresorptive drug, dose, duration of treatment and rate of development in patients receiving orthodontic treatment.

Condition being studied: Medication-related osteonecrosis of the jaw.

INPLASY registration number: This protocol was registered with the International Platform of Registered Systematic Review and Meta-Analysis Protocols (INPLASY) on 28 July 2020 and was last updated on 28 July 2020 (registration number INPLASY202070124).

\section{INTRODUCTION}

Review question / Objective: Is there any evidence that orthodontic treatment induces osteonecrosis of the jaws (ONJ) in patients treated with antiresorptive drug therapy? -Population: patients previously or under antiresorptive drugs and orthodontic treatment; -Interventions: any orthodontic treatment; -Comparison: patients undergoing orthodontic therapy not subjected to bisphosphonate therapy (risk assumed as negligible); -Outcome: state of knowledge regarding the risk of 
medication-related ONJ as it refers to type of antiresorptive drug, dose, duration of treatment and rate of development in patients receiving orthodontic treatment.

Rationale: The rationale of this systematic review is to appraise all data from relevant studies to determine whether orthodontic treatment induces osteonecrosis of the jaws (ONJ) in patients treated with antiresorptive drug therapy.

Condition being studied: Medicationrelated osteonecrosis of the jaw.

\section{METHODS}

Search strategy: The following four databases will be systematically searched; PubMed, EMBASE, Medline, and CINAHL. A comprehensive search strategy for all four databases has been developed as follows: 1. Bisphosphonate [MeSH Terms] OR diphosphonate [MeSH Terms] OR antiresorptive [MeSH Terms] denosumab [MeSH Terms] OR alendronic acid [MeSH Terms] OR zoledronic acid [MeSH Terms] OR pamidronate [MeSH Terms] OR etidronate [MeSH Terms] OR clodronate [MeSH Terms] OR ibandronate [MeSH Terms] OR risedronate [MeSH Terms] OR tiludronate [MeSH Terms] OR romosuzumab [MeSH Terms] 2 . Osteonecrosis [MeSH Terms] OR Avascular osteonecrosis [MeSH Terms] OR Osteonecrosis of the jaw [MeSH Terms] OR MRONJ [MeSH Terms] OR ONJ [MeSH Terms] OR BONJ [MeSH Terms] OR ARONJ [MeSH Terms] OR BRONJ [MeSH Terms] 3. Orthodontic [MeSH Terms] OR orthodontic appliance [MeSH Terms] OR lingual orthodontics [MeSH Terms] OR dental braces [MeSH Terms] OR orthodontia [MeSH Terms] OR teeth straitening [MeSH Terms] OR removable orthodontia appliance [MeSH Terms] OR fixed orthodontic appliance [MeSH Terms] OR malocclusion [MeSH Terms] OR teeth malposition treatment [MeSH Terms] OR orthodontia treatment [MeSH Terms] 4. 1 AND 2 AND 3 The search strategy will consider appropriate changes in the keywords and followed the syntax rules of each database.
Participant or population: Human participants with no restriction of age, gender, sample size or ethnic origin.

Intervention: Any orthodontic treatment.

Comparator: Patients not treated with medications associated with the development of MRONJ.

Study designs to be included: Randomized controlled trials, case-controlled trials, cohort studies (prospective and or retrospective), case series, retrospective studies analysis.

Eligibility criteria: Studies published or unpublished since 2003, systemic treatment with antiresorptive medication, orthodontic treatment to correct malocclusion.

Information sources: PubMed, EMBASE, Medline, and CINAHL.

Main outcome(s): To evaluate the current state of knowledge regarding the risk of ONJ as a result of antiresorptive drug therapy in patients undergoing orthodontic treatment for malocclusions.

Additional outcome(s): To determine whether the specific antiresorptive medication, duration of therapy, and indication for antiresorptive treatment contributed to adverse outcomes not limited to but including ONJ.

Data management: Following full text screening, data extraction will be completed using a predefined and standardized Microsoft Excel form. This will be done by two investigators independently. Any discrepancies will be resolved using a third investigator and conflicts resolved by discussion.

Quality assessment / Risk of bias analysis: Two review authors will independently appraise the risk of bias in the included studies. The authors will use the consensus-based clinical case reporting guidelines development (CARE) checklist for case reports and the Strengthening the 
Reporting of Observational Studies in Epidemiology (STROBE) checklist for case series and longitudinal studies. Any disagreements with the risk of bias assessments will be referred to a third author of the review team and subsequently resolved by discussion. Levels of evidence will be assessed according to the levels of evidence for therapeutic studies adapted from the American Society of Plastic Surgeons.

Strategy of data synthesis: Following a comprehensive screening to determine eligible studies, all selected papers will be carefully read to identify study and patient charactersitics. To assess primary and secondary outcomes, data will be extracted from each study and analysed. This will be done independently by two authors. Where pooling of results is inappropriate, the results will be reported as narrative descriptions using a detailed commentary.

Subgroup analysis: All participants will be included in the final analysis. If data permits, a subgroup analysis will be included in this review.

Sensibility analysis: If sufficient data are extracted, a sensitivity analysis will be conducted to check the stability of the outcome results by excluding low methodological quality or high risk of bias studies.

Language: No language restrictions will be applied.

Country(ies) involved: United Kingdom, Brazil.

Keywords: orthodontic treatment, orthodontist, osteonecrosis, MRONJ, tooth movement, antiresorptive drugs.

Dissemination plans: The systematic review will be submitted to a peer-reviewed journal for publication.

Contributions of each author:

Author 1 - Julian Woolley.

Author 2 - Oladapo Akintola.
Author 3 - Julian Yates.

Author 4 - Monica Diuana Calasans-Maia.

Author 5 - Jose de Albuquerque CalasansMaia.

Author 6 - Roberto Sacco. 